\title{
ENTWICKELT NOSEMA APIS ZANDER EINE RESISTENZ GEGENÜBER DEM ANTIBIOTIKUM FUMIDIL B ?
}

\author{
Nosema apis Zander développe-t-il une résistance à l'égard \\ de l'antibiotique Fumidil B? \\ K. P. GROSS und F. RUTTNER \\ Institut für Bienenkunde der Universität Frankfurt, Oberursel
}

SUMMARY

IS ZANDER'S NOSEMA APIS DISEASE IN BEES DEVELOPING A RESISTANCE

TO THE ANTIBIOTIC FUMIDIL B ?

By experimenting on bees in small cages we tried to discover whether continuous treatment with sublethal doses of Fumidil B can lead to the development of resistant strains of Nosema.

\section{Method}

Bees of the Carniolan variety hatched in the incubator were separated into cages in groups of 50 (fig. 1) and fed with one part syrup to one part water and with pollen paste. The infection was caught in the first two and a half days through bulk feeding with a standard suspension of spores (20 million spores per $\mathrm{ml}$ ). Each series of experiments was checked by a control group of noninfected bees. After infection and until the end of the experiment, on the twenty-first day, the bees were fed with Fumidil syrup in varying doses. A group of bees infected but not treated was kept as a control of the infective process. Each group of experiments consisted of five and sometimes ten repeats.

To interpret the results, the middle intestines taken from the bees were classed in five degrees of infection (classes 0 to 4 , fig. 2). For each group the percentage of bees affected and the average extent of infection in individual bees were calculated (Infection index) [1].

(1) The degrees of infection in different bees were added and the total divided by the number of bees examined in each group. The quotient is the index of infection. The percentage of bees affected in each group was calculated in the same way. 
During the attempt to secure power - of - resistance data, bees receiving weak doses of Fumidil were infected in ten sucessive test runs with a strain of Nosema which, to all appearances had never been in contact with this product (lenght of experiment : 14 months). In the next course the infection was obtained with the surviving spores from a previosly treated group. In the continuous experiment we always worked with three parallel doses of Fumidil and, according to the sensitivity of the infective agent, with doses of $1 / 2,1 / 4$ or $1 / 8$ of the normally prescribed dose $(10 \mathrm{mg}$, $5 \mathrm{mg}$ and. $2.5 \mathrm{mg}$ of active substance per litre of syrup) or with the full dose and doses of $1 / 2$ and $1 / 4$. Throughout the experiment the original material was allowed to multiply without treatment in order to provide matter for comparison which was fresh at the end of the trials.

\section{Results}

$1^{\text {o }}$ The observations of previous authors on the efficacy of treatment with Fumidil were confirmed (table 1). It is true that the infection has never been completely suppressed, even by large doses of Fumidil, but it has been abated to a very great extent. The toxic effect of high doses of Fumidil is made apparent by the shortening of the life-span.

$2^{\circ}$ In the continuous treatment with Fumidil B we noted that up to the third test run there was significant parallel growth of the infection in the three treated groups (for the half dose the proportion of bees affected went from $44 \%$ to $73 \%$ and to $86 \%$, the infection index from 0.2 to 2 and 2.6 . Tables 2 and 3 . Figures 3 and 4). After the fifth test run the infection decreased until at the tenth it reached a level significantly lower than that recorded at the beginning. After ten such runs of continuous treatment a comparison with the original untreated material shows that the agent has become less virulent, probably in proportion to toxic deterioration produced during sporogenesis. Variations in the strenght of the infection, at first inexplicable (e. g. during the eight test run) are probably due to complex corresponding factors between the host and the parasite.

Thus, during the continuous treatment, no resistant strain of Nosema appeared although during this time the number of spores formed was estimated at $10^{12}$. To explain the complex behaviour of the curve of infection the following theory can be put forward : a certain genetic potential exists for the formation of resistant forms during the test runs one to three. At the same time, a second mechanism intervenes to cause a cumulative toxic deterioration of the infective agent which at first can still be compensated on a genetic basis (test runs 4 to 6 ) but which finally leads to a general lowering of virulence (as also with the untreated control groups).

\section{ZUSAMMENFASSUNG}

In Käfigversuchen sollte festgestellt werden, ob eine Dauerbehandlung mit subletalen Dosen von Fumidil B zur Entstehung Fumidil-resistenter Nosemastämme führt.

\section{Methodik}

Im Brutschrank geschlüpfte Bienen der Carnica-Rasse wurden in Gruppen zu je 50 auf Käfige (Abb. 1) aufgeteilt und mit einer Zuckerlösung $1: 1$ und mit Pollenpaste gefüttert. Die Infektion erfolgte in den ersten zweieinhalb Tagen durch Massenfütterung mit einer standardisierten SporenSuspension (20 Millionen Sporen/ml). Bei jedem Versuchsansatz diente eine nichtinfizierte Gruppe als Kontrolle. Anschliessend wurde bis zur Auswertung des Versuches nach 21 Tagen mit einer Fumidil-Zuckerlösung verschiedener Dosierung gefüttert. Eine infizierte Gruppe blieb zur Kontrolle des Infektionsverlaufes unbehandelt. Jede Versuchsgruppe wurde in 5, in manchen Fällen in 10 Wiederholungen angesetzt.

Bei der Auswertung wurden die herauspräparierten Mitteldärme in 5 Befallsstufen (Klasse 0-4, Abb. 2) klassifiziert. Für jede Gruppe wurde der Prozentsatz befallener Bienen und die mittlere Befallsstufe der Einzelbienen (" Befall-Index ") berechnet.

Bei dem Versuch einer " Resistenzzüchtung " wurde derselbe Nosemastamm, der vermutlich noch nie mit Fumidil in Berührung gekommen war, durch 10 Bienenpassagen dauernd mit schwachen Fumidildosen behandelt (Versuchsdauer 14 Monate). Die Infektion der folgenden Passage erfolgte durch überlebende Sporen aus einer behandelten Gruppe der vorhergegangenen. Im Dauerversuch wurde immer mit drei Fumidildosierungen parallel gearbeitet und zwar je nach Empfindlichkeit des Erregers mit 1/2-facher, 1/4-facher und 1/8-facher Menge der vorgeschriebenen Dosis $(=10$ $\mathrm{mg}, 5 \mathrm{mg}$ und 2,5 mg Wirkstoff auf 1 Liter Zuckerlösung) oder mit einfacher, 1/2-facher und 1/4- 
facher Menge. Während der ganzen Versuchszeit wurde das Ausgangsmaterial ohne Bahandlung vermehrt, um zum Abschluss frisches Vergleichsmaterial zur Verfügung zu haben.

\section{Ergebnisse}

1. Die Angaben früherer Autoren über die Wirkung der Fumidilbehandlung wurden bestätigt (Tab. 1). Die Infektion wurde zwar auch bei hohen Fumidildosen nicht ganz unterdrückt, aber drastisch verringert. Die toxische Wirkung hoher Fumidildosen zeigte sich in einer Verkürzung der Lebensdauer.

2. Bei Dauerbehandlung mit Fumidil B war bis zur 3. Passage ein paralleles signifikantes An. steigen der Infektion bei allen 3 Fumidil-Versuchsgruppen festzustellen (bei 1/2-facher Dosierung stieg der Anteil der befallenen Bienen von 44 über 73 auf $86 \%$, der Befall-Index von 0,8 über 2,0 auf 2,6. Tab. 2 und 3, Abb. 3 und 4). Nach der 5. Passage fällt die Infektionsstärke bis zur 10. Passage bis unter die Ausgangswerte signifikant ab. Nach 10 Passagen Dauerbehandlung ergibt ein Vergleich mit dem unbehandelten Ausgangsmaterial, dass der Erreger weniger virulent geworden ist-vermutlich infolge toxischer Schädigung während der Sporogenese. Vorerst unerklärbare Schwankungen der Befallsstärke (z. B. in der 8. Passage) sind vermutlich Ausdruck einer komplexen Erreger-Wirtsbeziehung.

Es sind also während der Dauerbehandlung keine resistenteren Nosema-Stämme entstanden, obwohl während dieser Zeit schätzungsweise $10^{12}$ Sporen gebildet worden sind. Zur Erklärung des komplexen Verlaufs der Befallskurve wird folgende Hypothese vorgeschlagen : Es besteht ein gewisses genetisches Potential zur Ausbildung resistenterer Formen, die während der 1. bis 3 . Passage herausselektiert wurden. Gleichzeitig wirkt als 2. Mechanismus eine kumulative toxische Schädigung des Erregers, die zunächst auf genetischer Basis gerade noch kompensiert werden kann (4. bis 6. Passage), schliesslich aber zu einer allgemeinen Verminderung der Virulenz (auch bei unbehandelten Kontrollen) führt.

Die Nosematose verursacht in vielen - besonders in den nördlichen - Ländern mehr Völkerverluste als alle anderen Bienenkrankheiten zusammen. HIRSCHFELDER (1957) schätzte die Verluste in Bayern in den Jahren 1954-1956 auf 100000 Völker, das sind $20-25 \%$ des Bestandes. Im Lande Hessen wurden im gleichen Zeitraum für 4.179 Nosemafälle DM 142611 an Entschädigungen gezahlt. 1955 wurden von der Hessischen Tierseuchenkasse für Fälle der " Nosema-Amöbenseuche " DM 133901 entschädigt. Da nur seuchenhaft erkrankte Völker, die der Sachverständige abgeschwefelt hat, nicht aber abgestorbene Völker entschädigt werden, sind die tatsächlichen Verluste weit grösser. SvoвoDA schätzte 1957, dass in der CSSR $40 \%$ der Vökker erkrankt waren. Auch in der UdSSR treten nach Cebunin (1955) und GawriLow (1957) erhebliche Schäden durch Nosematose auf, in Waldbienenständen in der Tartarei in manchen Jahren bis zu $95 \%$ der Völker.

Die Ernteausfälle bei zwar überlebenden, aber im Frühjahr schwer angeschlagenen Völkern lassen sich noch viel schwerer schätzen als die Totalverluste. Sie sind in manchen Jahr aber sicherlich wesentlich grösser als diese. So wird sehr oft die Nosematose zum wichtigsten der begrenzenden Faktoren in der Honigproduktion.

Zur medikamentösen Therapie und Prophylaxe hat sich seit Jahrzehnten das Antibiotikum Fumagillin (Handelsbezeichnungen Fumidil B, Fumagillin) sehr gut bewährt (Katznelson und Jamieson, 1952; Hirschfelder, 1957; Steche, 1969). Der von Aspergillus fumigatus gewonnene Wirkstoff wurde zunächst in der Humanmedizin zur Bekämpfung von Entamöba histolytica angewendet, später jedoch wegen Nebenerscheinungen wieder fallengelassen.

Der Wirkungsmechanismus des Mittels gegen Nosema apis ist noch unklar. Fest steht, dass Fumagillin gegen den Erreger in seiner Sporenform unwirksam 
ist. Offenbar kommt Nosema apis erst dann in das "Schussfeld " dieses Antibiotikums, sobald der Parasit seine schützende Sporenhülle als Amöboidkeim verlassen hat und sich, leicht verwundbar, frei im Darmlumen bewegt. Es ist denkbar, dass der Parasit als Planont dann seine Beweglichkeit verliert und nicht mehr in der Lage ist, eine Darmepithelzelle zu infizieren. Es ist auch möglich, dass Fumagillin den Erreger erst in der späteren Phase in der Darmepithelzelle derart schädigt, dass es zu Störungen oder zum Erliegen der Zellteilungsvorgänge beim Erreger kommt. Diesen Eindruck konnten wir hin und wieder bei der Betrachtung der Mitteldärme von infizierten und mit Fumidil B behandelten Bienen gewinnen. Es schienen gelegentlich atypische Formen des Erregers aufzutreten.

Das Präparat Fumidil B steht dem Bienenzüchter nicht als Reinsubstanz zur Verfügung, sondern als ein Gemisch aus Wirkstoff, Puffer- und Trägerstoffen. In der käuflichen, lichtgeschützten braunen Flasche befinden sich in Pulverform 0,5 g Fumagillin-Wirkstoff als Bicyclohexylammoniumsalz; der Rest von etwa $24,5 \mathrm{~g}$ ist die Trägerstoff- und Puffersubstanz.

Zur Anwendung bei den Bienen wird nach Angaben des Herstellers $1 \mathrm{~g}$ Fumidilpulver in einem Liter einer Zuckerlösung (Verhältnis Zucker zu Wasser 1 : 1) eingerührt, beziehungsweise eine ganze Flasche Fumidil B in 25 Liter einer Zuckerlösung. Auf verschiedene Weise kann dann diese Lösung den Bienen zur Bekämpfung und Vorbeugung der Nosematose verabreicht werden. Am häufigsten wird im Frühjahr bei subakuter Nosematose eine Fütterung durch Futtergeschirre in Gaben von 1/4 bis 3/4 Liter pro Volk einige Tage lang durchgeführt. Bei akuter Nosematose erwies sich die Besprühung von Bienen und Waben am erfolgreichsten. Dabei lecken sich die durch die Krankheit schon angeschlagenen Bienen gegenseitig ab und kommen auf diese Weise noch mit dem Medikament in Berührung. Bei der Wintereinfütterung wird Fumidil B häufig vorbeugend verwendet. Ausser in Lösungen wird das Präparat auch erfolgreich im Futterteig, einem Honig-Puderzuckergemisch, verabreicht.

Es ist wiederholt vor einer generellen, prophylaktischen Fumagillinbehandlung gewarnt worden, weil die Gefahr einer Resistenzbildung beim Erreger und damit eines Verlustes der therapeutischen Wirksamkeit im Ernstfall bestände. Im Analogieschluss zum häufigen Auftreten resistenter Bakterienstämme im Verlaufe längerer Antibiotikabehandlungen wurde das Auftreten dieser Erscheinungen bei Nosema apis vielfach a priori stillschweigend vorausgesetzt. Es schien uns deshalb angezeigt, diese Frage an einem umfangreichen Material und bei einer ausreichenden Behandlungsdauer experimentell nachzuprüfen.

\section{MATERIAL UND METHODIK}

Aus Carnica-Völkern des Institutes, an denen bei Stichprobenuntersuchungen keine nosemainfizierten Bienen zu finden waren, wurden verdeckelte, reife Brutwaben entnommen und in einem sterilisierten Brutschrank bei $33-34^{\circ} \mathrm{C}$ und einer relativen Luftfeuchtigkeit von $70-80 \%$ bis zum Schlüpfen der Jungbienen untergebracht. Die frisch geschlüpften Bienen wurden in Gruppen zu je 50 auf sterilisierte Holzkäfige $(65 \times 65 \times 40 \mathrm{~mm}$, eine Breitseite mit Drahtgitter, eine mit Glas verschlossen; $A b b$. 1) verteilt. Anfangs wurden die Bienen einzeln abgezählt, später wurden die in einem Kühlschrank für kurze Zeit immobilisierten Bienen in einem geeichten Messzylinder abgemessen. Durch diese Vereinfachung wurde es möglich, mehr Versuchsgruppen und Wiederholungen unterzubringen.

Die Fütterung erfolgte mit einer Zuckerlösung $1: 1$ in einem $5 \mathrm{ml}$ fassendem Glasröhrchen 
und mit einer sterilen Pollenpaste ad libitum. Der Pollen wurde in den ersten 4, Tagen sehr stark, später kaum noch abgenommen. Die Bienen wurden ebenfalls bei einer Temperatur von $32-34^{\circ} \mathrm{C}$

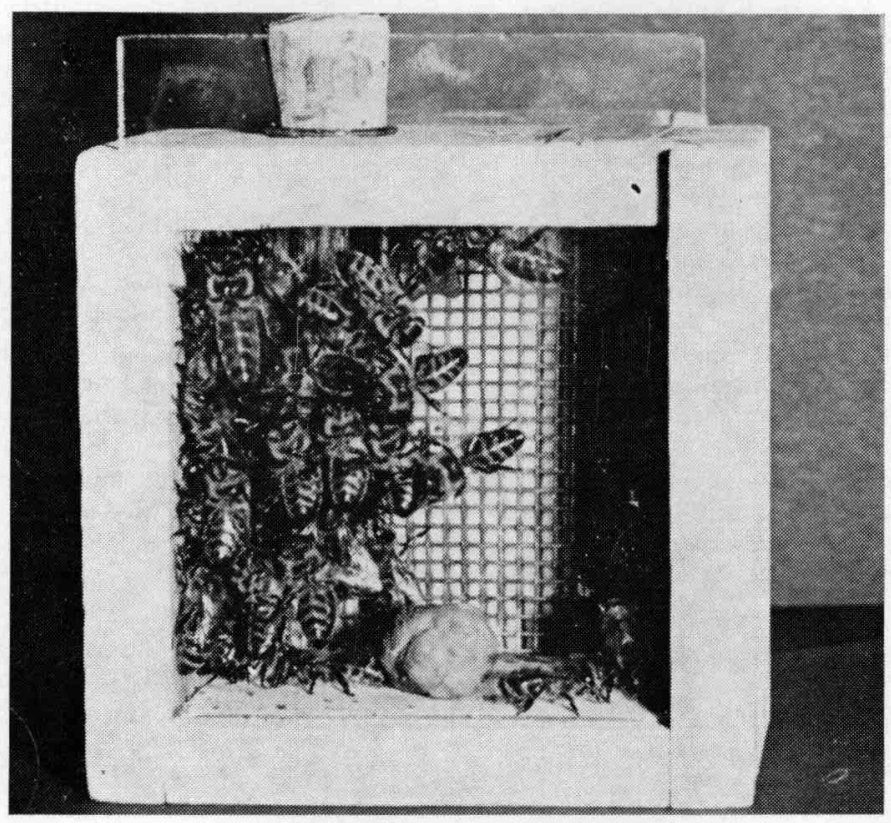

Авв. 1. - Versuchskäfig

FIG. 1. - Cagette expérimentale

und einer relativen Luftfeuchigkeit von 70-80\% gehalten. Die Käfige wurden mit einer Einlage aus Filtrierpapier zur Aufsaugung von austropfender Zuckerlösung und Kotflüssigkeit versehen.

Jeder Versuchsansatz wurde in 5-facher, in einigen Fälen in 10-facher Wiederholung durchgeführt.

Die zur Infektion bestimmten Versuchsbienen wurden in den ersten 2 1/2 Tagen mit einer Zuckerlösung gefüttert, dis pro Milliliter etwa 20 Millionen Nosemasporen enthielt. Dies entspricht einer mittelstarken Infektion.

Die Einstellung der Sporensuspension erfolgte zunächst mit Hilfe einer Zählkammer. Da sich aber herausstellte, dass die Zählung in einem Hämozytometer wegen der zu starken Flüssigkeitsschicht zu ungenau ist, da mehrere Sporen übereinander liegen und Pollenkörner sowie verschiedene Verunreinigungen stören können, wurde für den Dauerversuch folgendes Verfahren gewählt : Die Kotblasen und Mitteldärme der infizierten Bienen wurden in einem Mörser in Wasser zerrieben. Diese Sporenaufschwemmung wurde durch ein Haarsieb von grösseren Partikeln gereinigt und anschliessend in einem Becherglas für einige Stunden stehen gelassen, damit sich die Sporenmassen am Boden absetzen konnten.

In einer vorbereiteten Zuckerlösung $(1: 1)$ wurde ein Teil der Sporenmasse zunächst gleichmässig verrührt und davon ein Tropfen zwischen einen Objektträger und ein Deckgläschen gegeben. Unter dem Mikroskop wurde dann das Deckgläschen so stark aufgedrückt (dies lässt sich bei der klebrigen Zuckerlösung sehr gut durchführen), dass die in der Flüssigkeit treibenden Sporen gerade festgeklemmt wurden. Pollenkörner und andere Teile stören nicht, da sie zerdrückt werden bzw. immer neben die Sporen zu liegen kommen. Die Dicke der Sporen beträgt etwa $3 \mu$, die Grösse des Blickfeldes bei der gewählten Vergrösserung wurde berechnet, sodass der Rauminhalt auch errechnet werden konnte. Durch Auszählung von 50 Blickfeldern konnte die Sporenkonzentration bestimmt und gegebenenfalls die Suspension durch Zugabe von Sporen bzw. von Zuckerlösung auf 20 Millionen Sporen pro Milliliter eingestelft werden.

Das Infektionsmaterial musste aus einem Bienenvolk stammen, dass nicht schon vorher mit 
Fumidil in Berührung gekommen war. Wir holten deshalb Nosema-infizierte Bienen aus einem toten Volk von einem schon lange vernachlässigten und schliesslich verlassenen Bienenstand. Unmittelbar vor Beginn des Versuches wurde das Material in gekäfigten Bienen vermehrt.

In einem Vorversuch wurde festgestellt, dass diese Infektion durch Massenfütterung genau so gute Ergebnisse brachte wie die Einzelinfektion mittels einer Pipette. Auch bei individueller Fütterung gab es vereinzelt Bienen, die aus unbekannten Gründen nicht infiziert wurden. In einem weiteren Vorversuch wurde an 500 Bienen der Infektionsverlauf studiert : Beginnend am 3. Tag nach der Infektion zeigten sich kleine Infektionsherde im kaudalen Ende des Mitteldarmes; am 6. Tag zeigte der Darm in allen Abschnitten eine mittlere Infektionsstärke und am 9. Tag war der ganze Darm stark befallen.

Nach 2 1/2 Tagen wurde die Sporensuspension bei den unbehandelten Gruppen durch eine Zuckerlösung 1 : 1 ersetzt, bei den behandelten durch eine Fumidil-Zuckerłösung entsprechender Dosierung. Diese Fütterung wurde bis zum Abschluss des Versuchsansatzes (nach 20-22 Tagen) fortgesetzt. Legt man einen Nosema-Entwicklungszyklus von 4 Tagen zugrunde, so konnten sich in dieser Zeit 5 Generationen des Erregers entwickeln. Infizierte und nichtinfizierte Bienen wurden in getrennten Brutschränken gehalten.

Etwa 21 Tage nach Infektionsbeginn (in dieser Zeit hat die Sporenzahl nach Lotman 1944 ihr Maximum erreicht) wurden die überlebenden Bienen mit Schwefeldioxid abgetötet, der Mitteldarm wurde herauspräpariert und im Quetschpräparat unter dem Phasenkontrastmikroskop bei einer Vergrösserung 1 : 400 mit 30 Blickfeldern pro Biene der Befallsgrad ermittelt. Die Bienen, die nicht sofort ausgewertet werden konnten, wurden eingefroren und später untersucht.

Der Befallsgrad wurde nach einer Skala mit 5 Stufen ermittelt :

Авв. 2. - Klassifizierung der Sporendichte im Mitteldarm in 5 Stufen

Fıc. 2. - Schéma de classement de l'importance de l'infection selon cinq degrés

Stufe 0 : kein Befall

Degré 0 : aucune attcinte

Stufe 1 : schwacher Befall

Degré 1 : atteinte légère

Stufe 2 : mittlerer Befall

Degré 2 : atteinte moyenne

Stufe 3 : starker Befall

Degré 3 : forte atteintc

Stufe 4 : sehr starker Befall

Degré 4 : très forte atteinte
Es sind keine Nosema-Erreger im Blickfeld zu sehen

Pas de spores visibles dans le champ microscopique

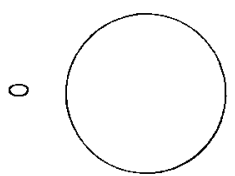

Bis etwa 10 Sporen pro Blickfeld

Environ 10 spores par champ

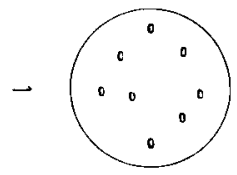

Bis etwa 50 Sporen pro Blickfeld

Environ 50 spores par champ

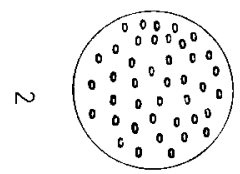

100-150 Sporen pro Blickfeld

100 à 150 spores par champ

Spore liegt an Spore

Spores très nombreuses

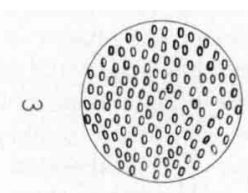

f

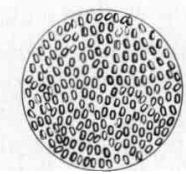

Die Befallsgrade der einzelnen Bienen wurden addiert und durch die Zabl der untersuchten Bienen pro Fragegruppe dividiert. Der Quotient ist der sogenannte Befall-Index $(=$ mittlerer Befallsgrad pro Biene). Weiterhin wurde der prozentuale Anteil der infizierten Bienen pro Fragegruppe errechnet. 


\section{ERGEBNISSE}

\section{1. - Prüfung des Wirkungsgrades von Fumidil B unter den gegebenen Laborbedingungen}

Die vom Hersteller empfohlene Dosis zur Bekämpfung und Vorbeugung der Nosematose ist $1 \mathrm{~g}$ Fumidil B auf 1 Liter Zuckerlösung (Verhältnis Zucker zu Wasser 1 : 1). Bezogen auf den reinen Wirkstoff (Aktivität) enthält demnach ein Liter Heilzuckerlösung $20 \mathrm{mg}$ Fumagillin-Wirkstoff. Nach den jahrelangen Erfahrungen vieler Institute und Imker ist diese Dosis in der Praxis im allgemeinen ausreichend, nosematöse Bienenvölker zu heilen bzw. einer Nosema-Erkrankung wirksam vorzubeugen.

Sehr wichtig war es, vor dem eigentlichen Hauptversuch die Frage zu klären, inwieweit das Medikament unter strengen Laborbedingungen in verschiedenen Konzentrationen in der Lage ist, eine künstliche, für Bienen tödliche NosemaInfektion bei gekäfigten Versuchstieren zu unterdrücken bzw. mit welcher Befallsstärke (Menge der überlebenden Erreger pro Biene) man bei Dauerbehandlung rechnen kann, um anschliessend weitere Passagen des Parasiten in neuen Versuchsserien zur Prüfung einer Resistenzbildung durchführen zu können. Hirschfelder (mündliche Mitteilungen) testete jährlich das auf dem Markt befindliche Fumidil B auf seine Wirksamkeit an gekäfigten, infizierten Bienen. Nach seinen Erfahrungen reicht unter den strengen Laborbedingungen bei starken künstlichen Infektionen die vorgeschriebene Dosis des Antibiotikums nicht aus, um alle Versuchsbienen nosemafrei zu halten. Zur erfolgreichen Bekämpfung der Nosematose bei freifliegenden Völkern wird jedoch auch von ihm diese Dosierung für ausreichend gehalten.

In unseren Vorversuchen wählten wir aus diesem Grund zur Dauerbehandlung infizierter Käfigbienen eine einfache, eine doppelte und eine vierfache Dosis Fumidil B, das heisst $20 \mathrm{mg}, 40 \mathrm{mg}$ und $80 \mathrm{mg}$ Aktivität pro Liter einer Zuckerlösung $1: 1$. Der Versuch wurde an 2500 Bienen durchgeführt. Es wurden fünf Gruppen mit je zehn Käfigen, die mit 50 im sterilen Brutschrank geschlüpften Bienen besetzt waren, aufgestellt und in der oben beschriebenen Weise gefüttert und infiziert.

Eine Gruppe (5) mit 10 Käfigen wurde nur mit reiner Zuckerlösung gefüttert und diente als nichtinfizierte, unbehandelte Kontrolle. Gruppen 1 bis 3 wurden anschliessend an die Infektion dauernd mit einer einfachen, doppelten und vierfachen Dosis von Fumidil B behandelt. Gruppe 4 wurde nach der Infektion mit reiner Zuckerlösung gefüttert.

Verwendet wurde ein Fumidil B der Firma Abbot (Lot Nr. 808-1407), dessen Wirksamkeit laut Aufschrift noch für 2 Jahre garantiert war.

Die Ergebnisse in Tabelle 1 zeigen, dass alle drei Fumidil B - Konzentrationen nicht ausreichten, eine Nosema-Infektion vollständig zu unterdrücken. Andererseits führt jedoch schon die einfache Dosis des Medikaments zu einer Senkung des Befall-Index von 3,95 (unbehandelte Kontrollen) auf 0,59. Bei diesem schwachen Infektionsgrad ist es nicht möglich, eine genügende Anzahl von Sporen zur Infektion neuer Bienengruppen für eine weitere Passage zu gewinnen. Um für die nächste Infektionsserie - es werden jeweils drei Fumidil-Gruppen und eine infizierte Kontrollgruppe benötigt - ausreichend viele Nosemasporen wiederzugewinnen, 
K. P. GROSS, F. RUTTNER

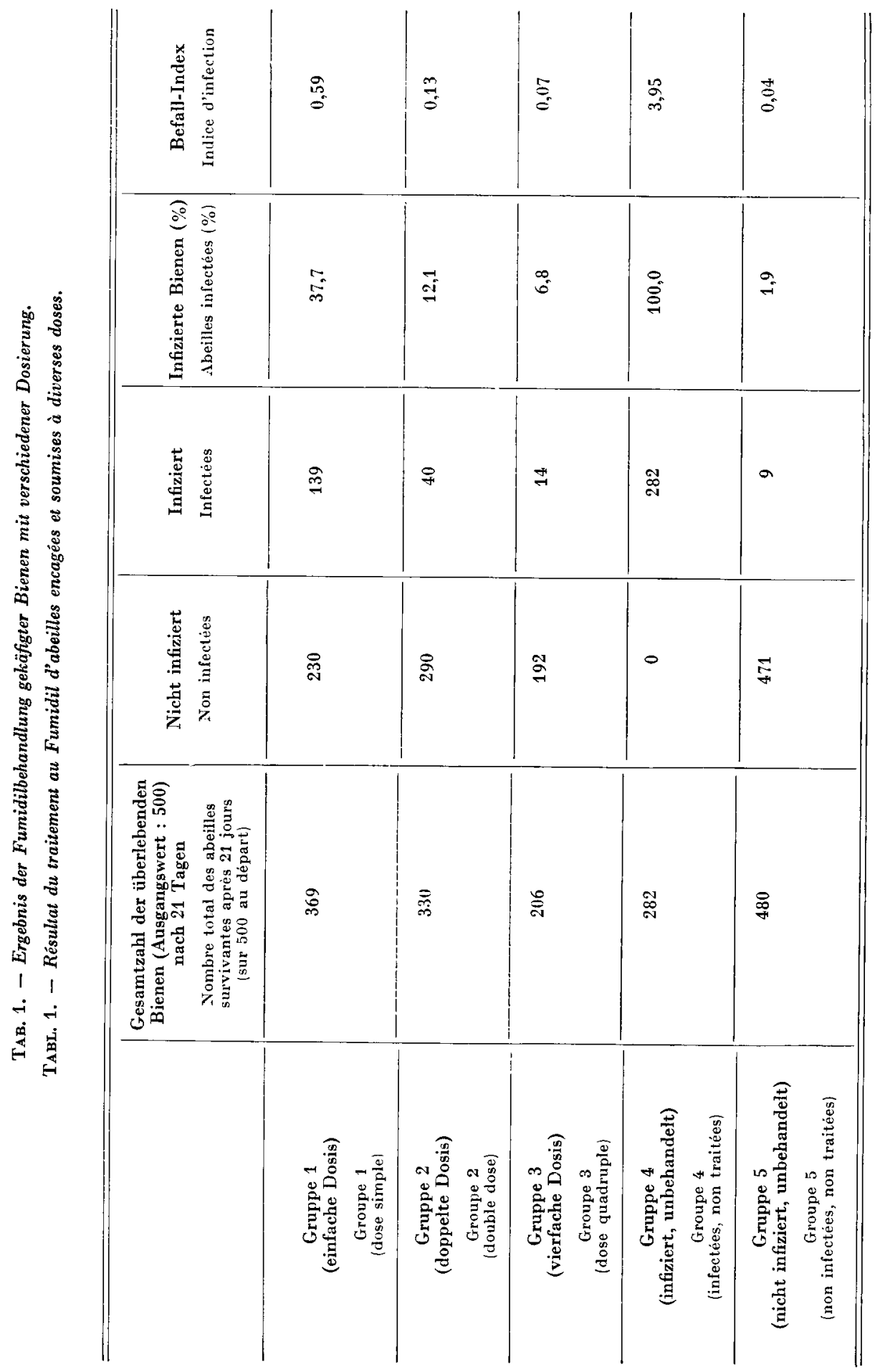


darf nach der Erfahrung unserer später durchgeführten Versuche (siehe weiter unten) in einer der Fumidil-Gruppen der Index von 2,0 nicht oder nur wenig unterschritten werden. Dies entspricht einer Reduktion der Anzahl der Erreger auf die Hälfte durch antibiotische Wirkung im Vergleich zur unbehandelten Kontrolle, deren Index etwa bei 4,0 liegt. Es werden etwa für jeden zu infizierenden Käfig während $21 / 2$ Tagen $10 \mathrm{ml}$ Sporensuspension benötigt, die pro ml etwa 20 Millionen Sporen enthalten soll. Der Futterverbrauch der Versuchsbienen ist zwar nicht so hoch, jedoch muss wegen rasch einsetzender Gärung im Futtergläschen die Sporenaufschwemmung zwei- bis dreimal erneuert werden.

Die Ergebnisse der Tabelle zeigen weiterhin, dass bei Gruppe 3 eine deutliche Lebensverkürzung eintritt, die sicherlich auf die Toxizität der vierfachen Fumidildosis zurückzuführen ist. An der Kontrollgruppe 4 ist ersichtlich, dass die Infektion einheitlich bei allen Bienen gut angegangen ist. Die nicht infizierte Gruppe 5, die in einem getrennten keimfreien Brutschrank gehalten wurde, blieb trotz aller Sorgfalt jedoch ebenfalls nicht ganz frei von Nosema-Infektionen.

Schwache Nosema-Infektionen der nichtinfizierten Kontrollgruppe wurden auch später einige Male beobachtet (Tab. 2, 3). Offenbar lässt sich nicht vermeiden, dass die Jungbienen von der Brutwabe manchmal eine geringe Zahl von Sporen in den Käfig schleppen, wo während der drei Wochen Inkubation eine Vermehrung und Verbreitung auf die angegebene Stufe stattfand. Da der spontane Infektionsgrad jedoch immer sehr niedrig blieb und da auf diesem Wege keine resistenten Formen eingeschleppt werden konnten, erfährt das Resultat dadurch keine Beeinträchtigung.

\section{2. - Versuch einer Züchtung eines Fumidil-resistenten Nosemastammes durch Dauerbehandlung mit subletalen Dosen}

Der Versuch wurde so angelegt, dass ein und derselbe Nosemastamm während 10 Passagen durch Bienen (das entspricht 50 Generationen des Erregers) ununterbrochen unter Fumidileinwirkung stand. Gleichzeitig wurde das Ausgangsmaterial unbehandelt vermehrt, sodass zum Abschluss der Versuche frisches Infektionsmaterial gleichen Ursprungs zur Verfügung stand, das aber noch nie mit Fumidil B in Berührung gekommen war.

Der Versuch erstreckte sich über mehr als ein Jahr (August 1968-September 1969). Die Methodik war im wesentlichen dieselbe wie oben beschrieben, doch erwiesen sich einige Modifikationen als notwendig :

\section{a. Gewinnung der Jungbienen.}

Um die Kontinuität zu sichern, mussten auch während der Wintermonate Jungbienen zur Verfügung stehen. Zu diesem Zwecke wurden im Herbst zwei Carnica-Völker in einem Raum mit annähernd konstanter Temperatur von $20^{\circ} \mathrm{C}$ gebracht. Es bestand Flugmöglichkeit ins Freie, aber nach der Methode von Louveaux war durch Anbringen eines Gittervorsatzes am Bienenkasten das Einströmen kalter Luft in das Volk verhindert. Durch regelmässige Fütterung von Pollen und Honig konnte die Brutpflege den ganzen Winter über in Gang gehalten werden. Im Frühjahr waren beide Völker erschöpft und es mussten z.T. auch andere Carnica-Völker zur Brutgewinnung heran* gezogen werden. Die verwendeten Bienen stammten also nicht aus demselben Volk, aber aus verwandten Linien derselben Rasse. Die Völker waren weder in der Saison vorher noch während des Versuches mit Fumidil B in Berührung gekommen. 
b. Fumidil-Dosierung.

Jeder Versuchsansatz (Passage) umfasste 5 Gruppen mit je 5 Käfigen zu 50 Bienen :

Gruppe 1- 3 : 3 verschiedene Fumidil-Dosierungen;

Gruppe 4 : Infiziert, nicht behandelt;

Gruppe 5 : Nicht infiziert, nicht behandelt.

Das Infektionsmaterial für sämtliche 4 Gruppen der nächsten Passage wurde jeweils von der Gruppe genommen, die einen Befall-Index von annähernd 2,0 aufwies. In dieser Gruppe war einerseits eine deutliche Fumidilwirkung vorhanden (Befall-Index auf die Häfte gesenkt), andererseits reichte die Sporenmasse für die neue Infektion.

Für den ganzen Versuch wurde dasselbe Fumidil-Präparat (Lot-Nummer A 813-253, Verfallsdatum 1-10-1969) verwendet. Es wurde ständig in festverschraubter Flasche im Kühlschrank bei $2-4{ }^{\circ} \mathrm{C}$ aufbewahrt.

Nach den Erfahrungen von Versuch 1) wurde für die erste Passage folgende Fumidildosierung gewählt :

Gruppe $1: 1 \mathrm{~g} /$ Liter (= $20 \mathrm{mg}$ Wirkstoff/Liter).

Gruppe $2: 0,5 \mathrm{~g} /$ Liter $(=10 \mathrm{mg}$ Wirkstoff/Liter).

Gruppe $3: 0,25 \mathrm{~g} /$ Liter (= $5 \mathrm{mg}$ Wirkstoff/Liter).

Das entspricht der einfachen, halben und Vierteldosis der vom Hersteller vorgeschriebenen Menge. Da sich diese Anfangsdosierung als etwas zu hoch erwies, musste sie in der zweiten Passage um eine Stufe gesenkt, ab der 3. wegen der veränderten Empfindlichkeit wieder gesteigert und ab der 7. Passage neuerdings gesenkt werden. Deshalb laufen nur die 1/2-fache und die 1/4-fache Dosis durch alle 10 Passagen (Tab. 2) .In der abschliessenden 10. Passage wurden alle vier Dosierungen eingesetzt (die 1/2-fache zur besseren statistischen Absicherung in 10, alle übrigen wie bisher in 5 Wiederholungen), ausserdem wurde das in der Zwischenzeit unbehandelte Ausgangsmaterial mit 1/2-facher Dosierung und unbehandelt getestet (Tab. 2, letzte Spalte). 
RESISTENZ GEGENÜBER FUMIDIL B.

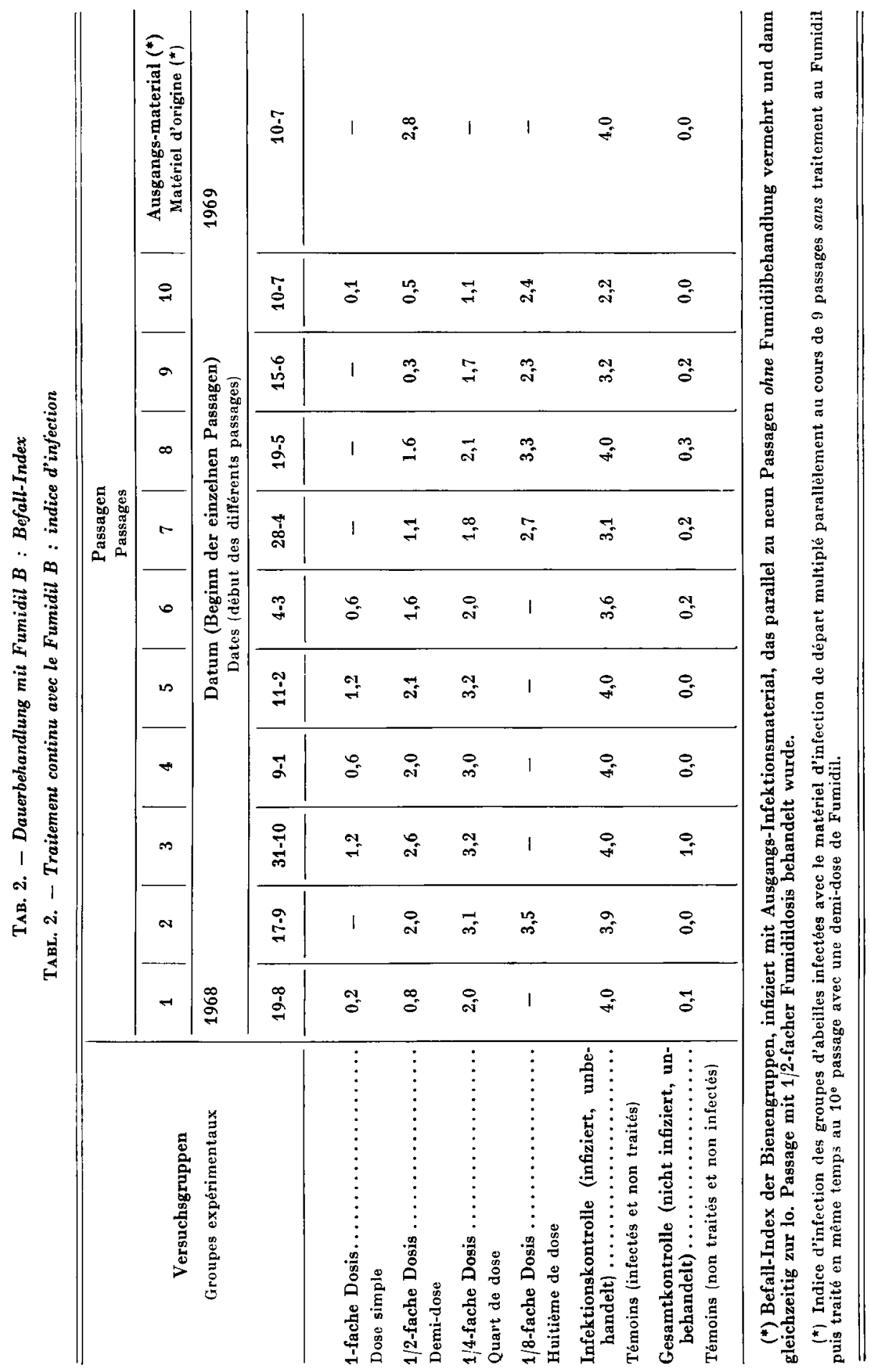


K. P. GROSS, F. RUTTNER

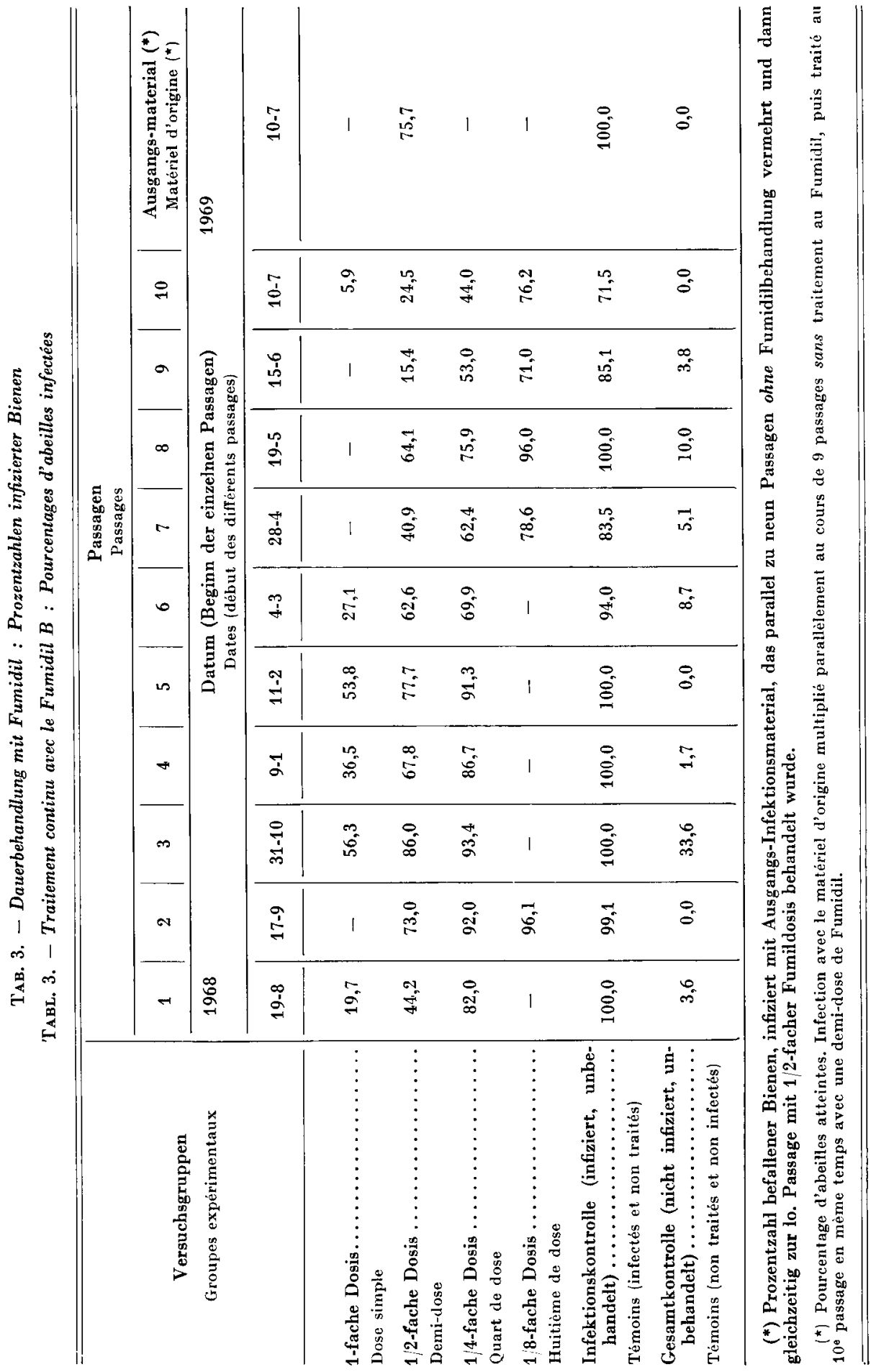


Befall - Index

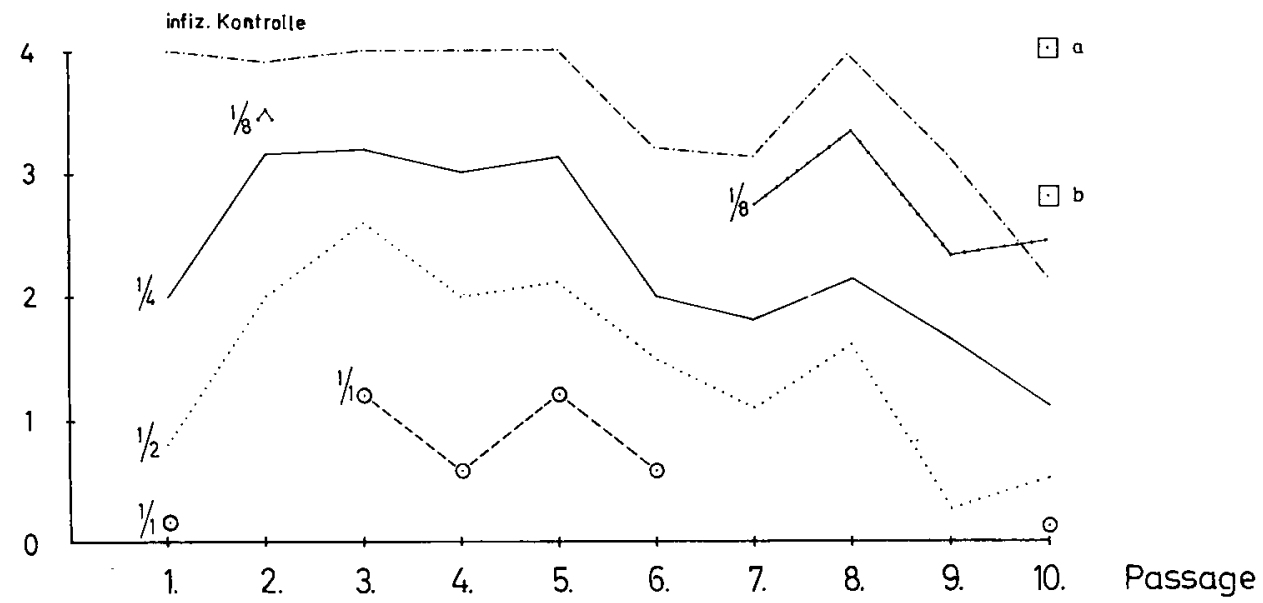

Авв. 3. - Befall-Index bei verschiedener Fumidil-Dosierung im Verlaufe von 10 Passagen

$1 / 1$ _ $\ldots \ldots$ Normaldosis ( 1 g Fumidil/1 Liter Lösung).

$1 / 2$. . . . . . . Halbe Dosis (=0,5 g Fumidil $/ 1$ Liter Lösung).

$1 / 4 \quad \ldots \ldots-\ldots-\ldots$ Vierteldosis (= $0,25 \mathrm{~g}$ Fumidil/1 Liter Lösung).

$1 / 8$. . . . . . . . Achteldosis (=0,125 g Fumidil $/ 1$ Liter Lösung).

_. . . . . . . . - Infizierte, unbehandelte Kontrolle.

a. Infektion mit Sporen des Ausgangsmaterials, das nie mit Fumidil in Berührung gekommen war, unbehandelt.

b. Wie a, aber Behandlung mit $1 / 2$ facher Fumidildosis.

Fig. 3. - Indice d'infection pour différentes doses de Fumidil au cours de 10 passages

$1 / 1$ - $\ldots \ldots$ Dose normale (= $1 \mathrm{~g}$ Fumidil 11 litre de solution).

$1 / 2$. . . . . . . . Demi-dose $(=0,5 \mathrm{~g}$ Fumidil $/ 1$ litre $)$.

$1 / 4$ - $\ldots \ldots \ldots$ Quart de dose $(=0,25 \mathrm{~g}$ Fumidil $/ 1$ litre $)$.

$1 / 8 .-.-.-., .-$. Huitième de dose $(=0,125 \mathrm{~g}$ Fumidil $/ 1$ litre $)$.

- . . . . . . . - Témoin infecté, non traité.

a. Infection avec spores du matériel de départ n'ayant jamais été en contact avec le Fumidif, non traité.

b. Comme $a$, mais traitement avec $1 / 2$ dose de Fumidif. 


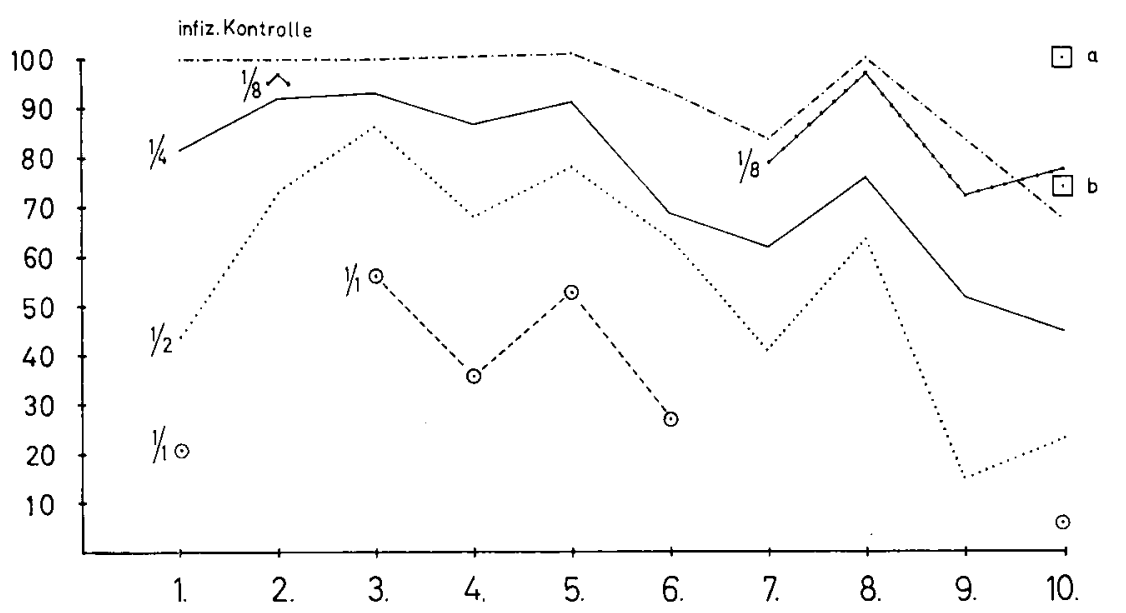

Aв8. 4. - Prozentsatz infizierter Bienen bei verschiedener Fumidil-Dosierung im Verlaufe von 10 Passagen Markierung der Kurven wie in Abb. 3

FIg.4. - Pourcentage d'abeilles infectées pour des doses diverses de Fumidil au cours de 10 passages Mêmes signes conventionnels que pour la figure 3

Aus den Versuchen (Tab. 2 und 3, Abb. 3 und 4) ergibt sich, dass es im Verlauf der zehn Passagen bei etwa 50 Generationen Nosema apis trotz Fumidil-Dauerbehandlung nicht gelungen ist, resistentere Erreger zu gewinnen.

Bis zur dritten Passage ist ein fast paralleles Ansteigen der Infektion bei allen Fumidilgruppen festzustellen. Während bei der 4. und 5. Passage die Werte ungefähr auf gleicher Höhe bleiben, fält die Infektionsstärke (allerdings mit einem deutlichen Wiederanstieg in der 8. Passage) bis zur 10. Passage wieder ab.

Nach 10 Passagen mit Fumidil-Behandiung war die Virulenz des Erregers signifikant geringer als das zur gleichen Zeit nochmals geprüfte Ausgangsmaterial. Das gilt für die mit Fumidil behandelten wie für die unbehandelten Gruppen. Ein so schlechtes Angehen der Infektion bei unbehandelten Bienen wie in der 2. Hälfte unseres Versuches war sonst nie zu beobachten.

Ermittelt man (durch Interpolation) die Fumidildosis, die zur Verringerung des Befall-Index auf die Hälfte, also auf 2,0 führt, so kann man eine Empfindlichkeitskurve des Erregers aufstellen (Abb. 5). Der Befall-Index 2,0 wird anfangs bei der 1/4-fachen Dosis erreicht, in der 3. Passage steigt die Konzentration auf die 3/4-fache Dosis, um bis zur 10. Passage fast auf die 1/8-fache abzufallen.

Für die statistiche Auswertung* der Ergebnisse wurden die Befall-Indices (der sogenannte " Gewogene Befall-Index ») zugrunde gelegt. Dabei wurden verteilungsunabhängige Rangteste (non-parametric) herangezogen bzw. die Vergleiche varianzanalytisch und mittels t-Test durchgeführt.

- Herrn Konrad Siller aus Ingelheim (Rhein) sind wir zu grossem Dank für die statistische Auswertung der Versuchsergebnisse verpflichtet. 
Die Versuchsgruppe mit einfacher Fumidildosis zeigt im Befall-Index von der 1. bis 3. Passage einen sehr signifikanten Anstieg $(p<0,001)$. 3., 4., 5. und 6. Passage sind einander statistisch gleichwertig $(p=0,05)$. Die 10. Passage ist sehr gesichert gegenüber 3 . und 6 . erniedrigt und gegenüber der 1 . Passage signifikant erniedrigt $(p<0,001$, bzw. $p<0,025)$.

Die Versuchsgruppe mit halbfacher Fumidildosis zeigt im Befall-Index von der 1. zur 2. Passage einen sehr signifikanten Anstieg, von der 2. zur 3. Passage einen signifikanten Anstieg $(p<0,0025$ bzw. $p<0,025)$ und nimmt sehr gesichert von der 3. bis 4. Passage wieder ab $(p<0,0005)$. Passage 4., 5. und 6. haben nur zufällig Unterschiede, der Abfall von 6 zu 7 ist schwach signifikant $(p<0,05)$, steigt dann wieder gesichert zur 8. Passage an $(p<0,01)$, sinkt höchst signifikant in der 9. $a b(p<0,0001)$. Der leichte Anstieg von der 9. bis 10. Passage ist statistisch gerade noch auffällig $(p<0,05)$. Der Befall-Index der 10. Passage ist gegenüber dem der 1. Passage hoch gesichert erniedrigt $(p<0,005)$.

Versuchsgruppe mit viertelfacher Fumidildosis : Die 2., 3., 4. und 5. Passage sind gleichwertig und gegenüber der 1. Passage sehr signifikant erhöht (1. Passage/5. Passage : $p<0,0025)$. Die 6. Passage ist gegenüber der 5. Passage signifikant erniedrigt $(p<0,025)$ und unterscheidet sich ansonsten gegenüber 7., 8 . und 9. Passage nur völlig zufällig, ebenso gegenüber der 1. Passage. Bei der 10. Passage ist der Befallindex gegenüber der 1 . Passage hoch gesichert vermindert $(p<0,0025)$.

Bei der Versuchsgruppe mit achtelfacher Fumidildosis haben die Passagen 2 und 8 einerseits und die Passagen 7, 9 und 10 andererseits nach statistischer Auswertung gleiche Befall-Indices. Die Passagen 2 und 8 sind jeweils hoch gesichert im Befall-Index erhöht gegenüber den Passagen 7, 9 und 10. (2. Passage/10. Passage : $p<0,0005)$.

\section{Dosis}

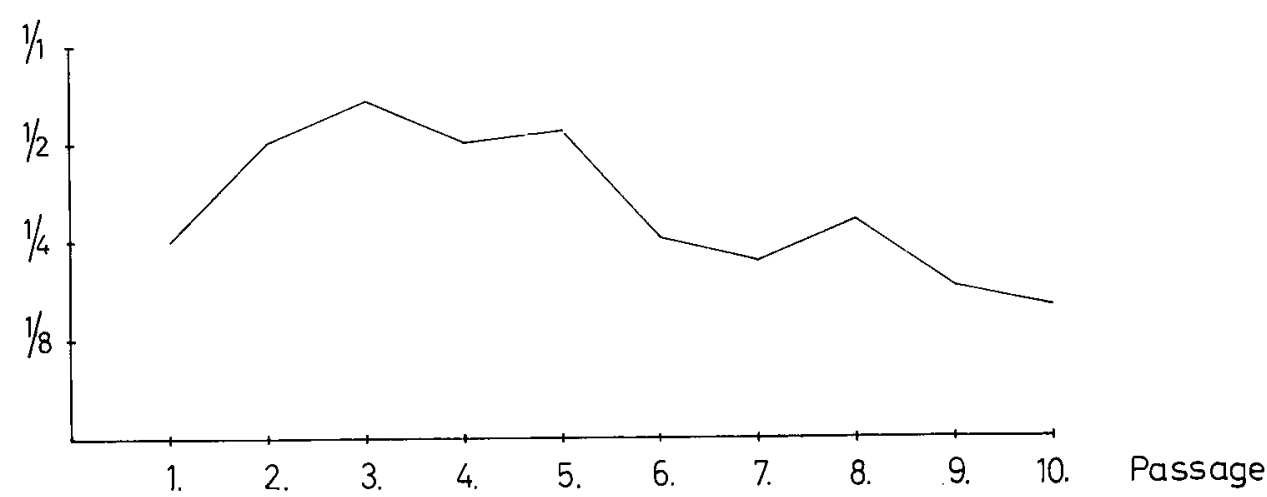

Aвв. 5. - Erforderliche Fumidildosis während der 10 Passagen um die Infektion auf einen durchschnittlichen Befall-Index von 2,0 zu senken

Fıg. 5. - Dose de Fumidil nécessaire pendant les 10 passages pour faire tomber l'infection à l'indice moyen de 2 
Die Passagen 1, 2, 3, 4, 5 und 8 der Infizierten Kontrolle (infizierte, nicht behandelte Bienen) haben den maximalen Befall-Index von 4,00. Bei der 6. Passage hat sich der Befall-Index signifikant $(p<0,025)$ gesenkt. Die 7. Passage ist gegenüber der 6. signifikant erniedrigt $(p<0,05)$, gegenüber dem maximalen BefallIndex von 4,00 sehr signifikant $(p<0,001)$. Die 7., 9. und 10. Passage unterscheiden sich nur zufällig in der Höhe des Befall-Index. Dass ab der 6. Passage die Infektion bei den Bienen der infizierten Kontrollen mit Ausnahme der 8. Passage nicht mehr so gut angegangen ist wie zum Beispiel bei den ersten fünf Passagen oder bei dem zuletzt parallel zur 10. Passage nochmals getesteten Ausgangsmaterial, fällt besonders ins Auge. Erstaunlicherweise konnte sich jedoch auch dort hin und wieder eine einzelne Biene absolut nosemafrei halten.

Eine Dosis-Wirkungsbeziehung lässt sich statistisch signifikant bis sehr signifikant innerhalb aller zehn Passagen feststellen, ausser bei der 6. Passage zwischen 1/2- und 1/4-facher Fumidildosis, bei der 7. Passage zwischen 1/8-facher Dosis und der infizierten Kontrolle sowie nicht in der 9. Passage zwischen 1/4-facher und 1/8-facher Fumidildosis und in der 10. Passage nicht bei 1/8-facher Dosis gegenüber der infizierten Kontrolle.

\section{DISKUSSION}

In Bakterienpopulationen treten primär- und sekundär-resistente Varianten in der Grössenordnung von $1: 10^{6}$ bis $1: 10^{8}$ (in Extremfällen bis $1: 10^{13}$ ) auf, das entspricht etwa der natürlichen Mutationsrate (WATTER u. Heilmeyer, 1969).

Die Bedeutung resistenter Varianten für die Therapie ergibt sich aus der Vererbbarkeit der Resistenzeigenschaft. Unter dem Selektionsdruck eines Chemotherapeutikums überleben nur die resistenten Keime und deren Nachkommen, sodass schliesslich eine resistente Population die Infektion in Gang hält, die dann zu Therapieversagern führen muss.

Bezogen auf die vorliegenden Versuche mit dem Erreger Nosema apis Z., der im Verlauf der 10 Passagen in etwa 50 Generationen trotz laufendem Fumidil"Beschuss" eine riesige Anzahl von Nachkommen hervorbringen konnte, kann festgestellt werden, dass bei diesem Infektionsmaterial keine resistentere Population entstanden ist. Bis zur 3. Passage deutet sich zwar eine Resistenzsteigerung durch paralleles Ansteigen der Infektion bei allen drei Fumidilversuchsgruppen an. Dieser Anstieg hielt jedoch nicht an, die Infektionsstärke blieb bei der 4. und 5 . Passage etwa auf gleicher Höhe stehen und fiel sogar bis zur 10. Passage bis unter die Ausgangswerte der 1. Passage wieder ab.

Geht man davon aus, dass man in stark infizierten Bienen, wie in der Literatur ziemlich übereinstimmend angegeben (GonTARSKI u. WAGNER, 1954; BorChert, 1966; Lotmar, 1940), ca. 180-500 Millionen Sporen findet, so sind bis zum Ende der Versuche schätzungsweise $10^{12}$ Erreger während fortwährender, ununterbrochener Fumidilbehandlung entstanden. Wäre ein Resistenz-Gen in der Population des Ausgangs-Infektionsmaterials vorhanden gewesen oder später entstanden, so hätte man bei einer so grossen Menge von Individuen, die sich trotz der fortlaufenden Behandlung mit unterschwelligen Fumidil-Dosen entwickelt hat, eine deutliche Resistenzentwicklung feststellen müssen. Der Grund für das Ausbleiben 
einer Resistenzentwicklung liegt daher offensichtlich nicht etwa an einer zu kleinen Zahl der in diesen Versuchen geprüften Nosema-Individuen, sondern in den besonderen biologischen Eigenschaften des benutzten Infektionsmaterials.

Es besteht somit kein Zweifel, dass das von uns benutzte Infektionsmaterial nicht in der Lage war, in dieser Zeit ein bestimmtes Gen zur Ausbildung einer Resistenz gegenüber dem Antibiotikum Fumidil B zu entwickeln. Im Gegenteil, die Fumidil-Empfindlichkeit schien zuzunehmen und ab der 6. Passage konnte sogar festgestellt werden, dass selbst in den infizierten Kontrollen, in denen das aus den vorhergegangenen Passagen stammende Sporenmaterial ohne neuerliche Fumidilbehandlung auf Infektiosität getestet wurde, die Stärke der Infektion absank.

Die Kurve der infizierten Kontrolle läuft — statistisch sicher — nahezu parallel mit den Kurven der einzelnen Fumidilgruppen. Die Dosiswirkungsbeziehung war sehr signifikant. Die in der Untersuchung gefundenen Werte variieren also nicht unabhängig voneinander, sondern stehen in einem bestimmten Verhältnis zueinander. Die Wirkung des Heilmittels blieb demnach bei allen Passagen gleich, nur die Infektiosität der Erreger änderte sich.

Es handelt sich aufgrund der statistischen Auswertung nicht um eine zufällige Erscheinung, dass sich bei den letzten Passagen die Nosemaerreger deutlich weniger infektiös erwiesen. Für den Abfall der Infektionsstärke gegen Ende der Versuchsserien sind zwei Erklärungen möglich :

1. Ein Unterschied im physiologischen Zustand der Versuchsbienen im Laufe des Jahres, der zu einer unterschiedlichen Abwehrkraft führt. Denn als Versuchsbienen für die 3., 4. und 5. Passage mussten Winterbienen genommen werden. Von diesen weiss man, dass sie besonders widerstandsfähig gegen Krankheiten, auch gegen Nosematose sind. Dies kommt auch im Saisonverlauf der Nosematose zum Ausdruck (Borchert, 1928; GoETZE et al., 1959; BAILEY, 1963). In unseren Versuchen konnte jedoch zu diesem Zeitpunkt keine Verringerung, sondern sogar eine deutliche Steigerung der Infektionsstärke beobachtet werden. Während im natürlichen Nosemaverlauf im März, April und Mai immer eine Steigerung der Befallstärke bei freifliegenden Bienenvölkern in der Natur auftritt, ist hier während dieser Zeit (6. und 7. Passage) eher eine,Verringerung der Infektionsstärke festzustellen. Der jahreszeitlich bedingte physiologische Zustand der Bienen kann demnach nicht der Grund für den schwankenden Verlauf der Infektionskurven im Verlauf eines ganzen Jahres (von August 1968 bis Juli 1969) sein. Auch spricht weiterhin gegen diese mögliche Erklärung, dass die Versuchsbienen der 10. Passage genauso Juli-Bienen waren, wie die zur Prüfung des Ausgangs-Infektionsmaterials gleichzeitig zu dieser 10. Passage verwendeten Versuchsbienen und sich dennoch ein erheblicher Unterschied in der Infektionsstärke zwischen beiden Gruppen zeigte.

Lediglich der Anstieg der Infektion in allen Gruppen der 8. Passage (Infektionsbeginn am 19.5., zum Zeitpunkt des Infektionsmaximums in den Völkern) könnte vielleicht auf diese Weise erklärt werden.

2. Eine Änderung der Virulenz durch die Dauerbehandlung mit Fumidil B, also zum Beispiel eine Schädigung des Erregers in seinem Stoffwechsel durch dieses Antibiotikum während der Sporogenese. Hier muss nochmals darauf hingewiesen werden, dass der Befall-Index in den letzten Passagen bei den infzierten. 
unbehandelten Kontrollen signifikant abnimmt, während das gleichzeitig nochmals geprüfte Ausgangsmaterial bei den infizierten Kontrollen dasselbe Ergebnis wie anfangs brachte. Gleichzeitig ist bei diesen Passagen die Dosiswirkungsbeziehung nicht so deutlich wie zu Beginn des Versuchs. Es ist wahrscheinlich, dass sich darin eine fortdauernde Schädigung des Erregers durch Fumidil B zu erkennen gibt. Demgegenüber ergibt sich in den ersten drei Passagen ein bei allen Dosierungen gleichlaufendes und statistisch hoch signifikantes Ansteigen des Befall-Index. Für diesen gegensinnigen Verlauf der Befallskurve während des Gesamtversuchs bietet die Annahme von zwei, voneinander unabhängigen Wirkungsmechanismen die naheliegendste Erklärung : Zuerst erfolgt eine Selektion resistenterer Formen, gleichzeitig wird der Erreger durch die Dauerbehandlung kumulativ geschädigt bis zu einer Stufe, auf der eine genetische Kompensation durch Selektion nicht mehr möglich ist. Von diesem Zeitpunkt an (5. Passage) nimmt die Virulenz des Erregers ab. Eine Ausnahme bildet die achte Passage, die zeitlich zusammenfällt mit dem Befallsmaximum im freifliegenden Bienenvolk.

Für diese Annahme spricht auch, dass gelegentlich während der mikroskopischen Untersuchung der Eindruck bestand, dass atypische Formen des Erregers auftraten, Riesen- und Zwergformen sowie Missbildungen des Zellleibes im Vergleich zu den unbehandelten Nosemaerregern in den Kontrollbienen.

Trotzdem ergaben sich im Verlaufe dieser Versuche einige Nebenbefunde, die sich nicht ohne weiteres erklären lassen. Wie ist es zu dem neuerlichen Ansteigen der Infektion in allen Gruppen während der 8. Passage gekommen ? Und wie ist es zu erklären, dass die Behandlung mit der 1/2-fachen Dosis während der 1. Passage (19-8-1968) zu 44,2\% infizierten Bienen mit einem Befall-Index von 0,8 geführt hatte, während dieselbe Dosis bei demselben, inzwischen ohne jede Behandlung vermehrten Material ein Jahr später (10-7-1969) die Infektion nur auf 75,7 \% der Bienen und einen Befall-Index von 2,8 senkte ? Möglicherweise kommt hier eine komplexe Erreger-Wirtbeziehung zum Ausdruck, die nur mit anderen Methoden analysiert werden könnte.

Nach der Hypothese STEches (1965) vom Ablauf zweier Entwicklungszykden mit der sogenannten "Aktivphase " im Frühjahr und Sommer sowie den sogenannten "Ruhestadien " des Erregers im Herbst und Winter mit einem Absinken der absoluten Anzahl fertiger Sporen in dieser Zeit hätten auch in unseren Versuchenunter der Voraussetzung, dass die Verhältnisse bei gekäfigten Bienen mit denen im freifliegenden Volk vergleichbar sind - während der Wintermonate (3., 4. und 5. Passage) die Zahl der Nosemasporen in den einzelnen Gruppen deutlich abnehmen und sich die Erreger als "Kernbefallsstadien " dem Blick entziehen müssen. Das war nicht der Fall. Wie bereits oben geschildert, war gerade während dieser Zeit die grösste Befallsstärke mit Nosemasporen des ganzjährig durchgeführten Versuchs festgestellt worden, die dann in den folgenden Frühjahrsmonaten sogar stetig abfiel.

Bei dem für den Menschen pathogenen Protozoon Entamoeba histolytica konnte NaKamura (1961) durch Fumagillinbehandlung in geringen Konzentrationen plötzlich hohe Resistenzgrade hervorrufen. Wäre ein Analogieschluss erlaubt, hätte man bei unseren Versuchen auch innerhalb kurzer Zeit hochgradig resistente Varianten von Nosema apis erwarten müssen. Aber bei den beiden Erregern handelt es sich schliesslich um zwei völlig verschiedene Protozoen mit 
anderer Wirtsbindung, nämlich menschenpathogene Rhizopoden und insektenpathogene Mikrosporidier.

Bei unseren Versuchen zur Frage der Resistenzbildung handelte es sich um Untersuchungen unter extremen Laborbedingungen. In der praktischen Imkerei wird es kaum vorkommen, dass ununterbrochen über ein ganzes Jahr hinweg eine Fumidilbehandlung vorgenommen wird. Höchstens während weniger Wochen, oft nur an einigen Tagen im Jahr wird eine Behandlung am Bienenvolk durchgeführt, im Vergleich zum geschilderten Versuch nur eine kleine Zeitspanne. In der Zwischenzeit könnten sich dann unzählig viele Generationen Nosema apis entwickeln, ohne mit Fumidil B in Berührung zu kommen. Allein aus diesem Grunde kann sich im praktischen Imkerbetrieb eine Resistenz des Erregers kaum entwickeln. Wie die vorliegende Untersuchung gezeigt hat, ist aber selbst bei Dauerbehandlung nicht mit dem Auftreten fumagillinresistenter Formen zu rechnen.

Misserfolge bei der Anwendung von Fumidil B in der praktischen Imkerei, die hin und wieder vorkommen, sind vorwiegend auf Reinfektionen oder auch auf fehlerhafte Anwendung zurückzuführen, z.B. übermässige Erwärmung oder zu langes Stehenlassen der Heillösung unter Licht- und Sauerstoffzutritt, sodass es zu einem Zerfall des Wirkstoffes kommen kann. Oft können die vorwiegend erkrankten Flugbienen bei den üblichen Therapiemethoden, Fütterung mittels Futtergeschirren oder Futterteig, nicht genügend Heiłsubstanz aufnehmen. Man sollte daher dem Fumidil-Sprühverfahren den Vorrang geben, mit dem bei eingestelltem Flugbetrieb auch die Flugbienen gut zu erreichen sind. Es ist auch denkbar, dass bei bereits zu stark nosemageschädigten Bienenvölkern und sehr schlechten Witterungsbedingungen die Hilfe zu spät kommt. Auch Überwinterung auf Honigtau, die bei den Völkern Ruhr zu Folge hat, und Mischinfektionen mit Malpighamöben sowie auch Septikämieen und Rickettsiosen können einige der Gründe sein, die ein Versagen der Wirkung des Fumidil B gegen Nosema apis Z. vorgetäuscht und zur Annahme einer " bereits erfolgten Resistenz " geführt haben.

Eingegangen im Juli 1970.

Reçu pour publication en juillet 1970.

\section{RÉSUMÉ}

Par des expériences conduites en cagettes on a cherché à savoir si un traitement continu des abeilles avec des doses sublétales de Fumidil B peut conduire à l'apparition de souches résistantes de Noséma.

\section{MÉTHODE}

Des abeilles de race carniolienne écloses à l'étuve ont été réparties par groupes de 50 dans des cagettes (fig. 1) et nourries avec un sirop de sucre $1: 1$ et une pâte de pollen. L'infection était obtenue dans les deux premiers jours et demi par un nourrissement massif au moyen d'une suspension standard de spores (20 millions de spores par $\mathrm{ml}$ ). Pour chaque série d'expériences on disposait d'un groupe témoin constitué par des abeilles non infectées. Après l'infection et jusqu'à la fin de l'expérience, le $21^{\mathrm{e}}$ jour, on nourrissait les abeilles avec un sirop au Fumidil à des doses diverses. Un groupe d'abeilles infectées et non traitées était conservé comme témoin du processus d'infection. Chaque groupe d'essais comportait 5 et parfois 10 répétitions. 
Pour l'interprétation des résultats on a classé les intestins moyens prélevés sur les abeilles seion 5 degrés d'infection (classes 0 à 4 , fig. 2). Pour chaque groupe on a calculé le pourcentage d'abeilles atteintes et le degré moyen d'infection des individus (indice d'infection) (1).

Au cours de l'essai d'obtention d'une résistance, on a traité par 10 passages successifs sur abeilles recevant de faibles doses de Fumidil une souche de Nosema qui, selon toute vraisemblance, n'avait jamais été en contact avec ce produit (durée de l'expérience : 14 mois). L'infection au passage suivant était obtenue avec les spores survivantes provenant d'un groupe précédemment traité. Dans l'essai continu on a toujours travaillé avec trois doses parallèles de Fumidil et, selon les sensibilités de l'agent infectieux, avec des doses $1 / 2,1 / 4$, ou $1 / 8$ de la dose normalement prescrite (soit $10 \mathrm{mg}, 5 \mathrm{mg}$ et $2,5 \mathrm{mg}$ de substance active pour un litre de sirop de sucre) ou bien avec la dose simple et les doses $1 / 2$ et $1 / 4$. Pendant toute la durée de l'expérience le matériel de départ fut multiplié sans traitement de façon à fournir à la fin des essais un matériel de comparaison qui soit frais.

\section{RÉSULTATS}

1. Les observations antérieures d'auteurs sur l'efficacité du traitement au Fumidil ont été confirmées (tab. 1). L'infection n'a jamais été, il est vrai, totalement supprimée, même par l'application de fortes doses de Fumidil, cependant elle a été diminuée dans de très fortes proportions. L'effet toxique des hautes doses de Fumidil s'est manifesté par un raccourcissement de la durée de vie.

2. Dans le traitement continu au Fumidil $B$ on a noté jusqu'au $3^{e}$ passage une croissance parallèle significative de l'infection dans les trois groupes traités (pour la demi-dose la proportion des abeilles atteintes passe de 44 à 73 et $86 \%$, l'indice d'infection de 0,8 à 2 et 2,6 . Tab. 2 et 3 . fig. 3 et 4). Après le $5^{\mathrm{e}}$ passage le pouvoir infectieux décroît jusqu'au $10^{\mathrm{e}}$ passage pour atteindre un niveau inférieur à celui qui était constaté au départ, et ceci de façon significative. Après 10 passages de trai. tement continu une comparaison avec le matériel de départ non traité montre que l'agent est devenu moins virulent, probablement en raison de détériorations d'origine toxique se produisant au cours de la sporogénèse. Les variations tout d'abord inexplicables de la force de l'infection (par exemple au

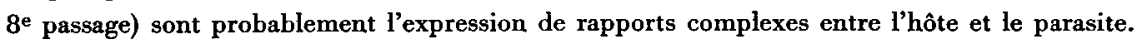

Donc, pendant le traitement continu, on n'a vu apparaître aucune souche résistante de Nosema bien que pendant cette période on puisse évaluer à $10^{12}$ le nombre de spores formées. Pour expliquer l'allure complexe de la courbe d'infection on peut avancer l'hypothèse suivante : il existe un certain potentiel génétique pour la formation de formes résistantes qui, pendant les passages 1 à 3 se trouvent sélectionnées. En même temps, un second mécanisme intervient pour provoquer une détérioration toxique cumulative de l'agent infectieux qui, tout d'abord, peut encore être compensée sur une base génétique (passages 4 à 6 ) mais qui conduit finalement à une baisse générale de la virulence (aussi chez les témoins non traités!).

\section{LJTERATURVERZEICHNIS}

BatLey L., 1963. Infectious diseases of the honey bee. Land Books, London.

Borchert A., 1928. Beiträge zur Kenntnis des Parasiten Nosema apis. Archiv Bienenkunde, Ix, 115117.

Borchert A., 1966. Krankheiten und Schädlinge der Honigbiene. S. Hirzel Verlag, Leipzig.

Cebunin G. J., 1955. Zit. nach Kettner, 1958.

(1) Les degrés d'infection des différentes abeilles sont additionnés et le total obtenu est divisé par le nombre des abeilles examinées dans chaque groupe. Le quotient est l'indice d'infection. On calcule également le pourcentage des abeilles atteintes dans chaque groupe. 
GavriLov B. N., 1957. Zit. nach Kettner, 1958.

Goetze G., Eberhard F., Zeutschel B., 1959. Versuche zur Selbstheilung und Therapie der Nosematose der Honigbiene. Aus dem Institut f. Bienenkunde der Rheinischen FriedrichWilhelms-Universität Bonn.

Gontarski H., Wagner O., 1954. Quantitative Versuche zur chemotherapeutischen Bekämpfung von Nosema apis Zander bei der Honigbiene. Arzneim. Forsch. 4, 161-168.

Hirschfelder H., 1957. Alte und neue Erfahrungen zur Nosema-Bekämpfung. Mitt. Bayr. Landes anstalt $f$. Tierzucht, 65-68.

Katznelson H., Jamieson C., A., 1952. Control of Nosema disease of honey bees with fumagillin. Science, 115, 70-71.

Ketrner H., 1958. Beiträge zum Nosemaproblem der Bienen. Anchiv Geflügelzucht Kleintierkunde, VII, 326-345.

Lotmar R., 1940. Beiträge zur Pathologie des Bienendarmes (Apis mellifica). Ǔber den Infektionsverlauf und die Vermehrung des Parasiten Nosema apis. Landwirtsch. Jahrbuch d. Schweiz, Verbandsdruckerei, Bern.

Lotmar R., 1944. Uber den Einfluss der Temperatur auf den Parasiten Nosema apis. Beihefte Schweiz. Bienenztg., 1/6, 262-284.

Steche W., 1965. Zur Ontogenie von Nosema apis Zander im Mitteldarm der Honigbiene. Bull. Apicole, 8, 181-212.

Steche W., 1969. Die Abhängigkeit der Entwicklung des Parasiten Nosema apis (Zander) vom Brutverlauf im Bienenvolk. Verh. XXII. Int. Kongr. Bienenzu:ht, 583-585.

Svoвоda J., 1957. Der Stand der Bienenkrankheiten in der Tschechoslowakei. Leipziger Bienenztg., 71, 152-153.

Walter A. M., Heilmeyer L., 1969. Antibiotika-Fibel (3. Auflage). Georg Thieme Verlag, Stuttgart.

Nakamura M., 1961. Induced increase in resistance of Entamœba histolytica to Fumagillin. Nature, 191, 413-414.

Anschrift der Verfasser :

Dr. K. P. Groß

6 Nieder Eschbach über Frankfurt/Main

Friedenstrasse 19
Prof. Dr. Dr. F. Ruttner

Institut für Bienenkunde 637 Oberursel Ts. Im Rosengärtchen 